\title{
白苞裸蒴中两个新的含氮类化合物
}

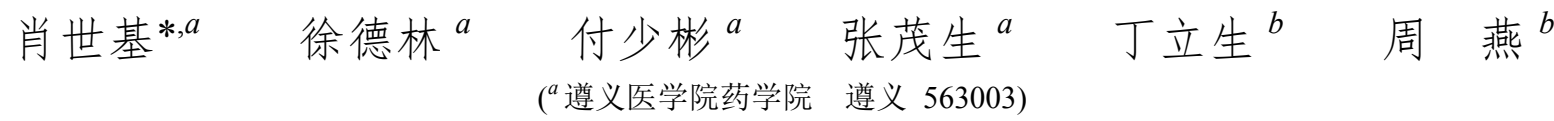 \\ ( ${ }^{b}$ 中国科学院成都生物研究所 成都 610041)
}

\begin{abstract}
摘要 通过硅胶和 MCI 柱色谱以及制备液相色谱等分离方法从白苍裸蒴中分离纯化得到 6 个含氮类化合物, 经质谱和 核磁共振等波谱技术将其结构分别鉴定为 2-(1-差基-6-甲氧基-4-氧亚基环己-2-烯-1-基)乙腈(1)、10-甲氧基-1-氧杂-3-氮 杂螺 $[4.5]$ 癸-2,6-二烯-8-酮(2)、2-(1-羊基-4-氧亚基环己-2,5-二烯-1-基)乙腈(3)、1-氧杂-3-氮杂螺[4.5]癸-2-烯-8-酮(4)、1氧杂-3-氮杂螺 $[4.5]$ 癸-2,6-二烯-8-酮(5)、对羟基苯乙腈(6). 化合物 $\mathbf{1}$ 和 $\mathbf{2}$ 为新的含氮类化合物, 化合物 $\mathbf{3}$ 为新的天然产 物, 其余化合物均为首次从该植物中分离得到.
\end{abstract}

关键词 白苞裸蒴; 三白草科; 含氮类化合物; 化学成分

\section{Two New Nitrogen Compounds from Gymnotheca involucrata}

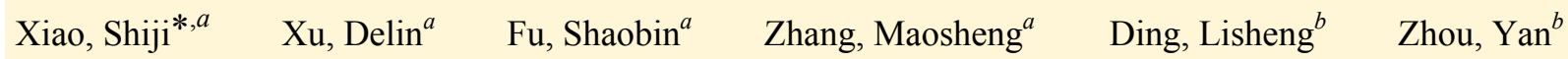 \\ ( ${ }^{a}$ School of Pharmacy, Zunyi Medical University, Zunyi 563003) \\ ( ${ }^{b}$ Chengdu Institute of Biology, Chinese Academy of Sciences, Chengdu 610041)
}

\begin{abstract}
By silica gel, MCI column chromatographic and preparative HPLC (high performance liquid chromatography) technologies, six nitrogen compounds were isolated from the whole parts of Gymnotheca involucrata. By using spectroscopic techniques including NMR and MS, these compounds were identified as 2-(1-hydroxy-6-methoxy-4-oxocyclohexa-2-ene1-yl)acetonitrile (1), 10-methoxy-1-oxa-3-azaspiro[4.5]dec-2,6-diene-8-one (2), 2-(1-hydroxy-4-oxocyclohexa-2,5-dien-1-yl)acetonitrile (3), 1-oxa-3-azaspiro[4.5]dec-2-ene-8-one (4), 1-oxa-3-azaspiro[4.5]dec-2,6-diene-8-one (5) and 4-hydroxybenzyl acetonitrile (6). Compounds $\mathbf{1}$ and $\mathbf{2}$ are new compounds, compound $\mathbf{3}$ is a new natural product, and compounds $\mathbf{4} \sim \mathbf{6}$ were isolated from this plant for the first time.
\end{abstract}

Keywords Gymnotheca involucrata; saururaceae; nitrogen compound; chemical constituent

白苍裸蒴(Gymnotheca involucrata Pei)为三白草科 中我国特有裸蒴属的草本植物, 在贵州又名白折耳, 水 折耳. 白苞裸蒴主要产于我国四川南部的峨眉山和贵州 东南部等地, 生于海拔 1000 米左右的路旁或者林中湿 地上 ${ }^{[1]}$. 《中国中药志要》记载其全草辛, 温, 清热利湿, 活血化瘀，止带. 用于跌打损伤，肺疼咳嗽，白浊，带下 病和腹胀水肿 ${ }^{[2]}$. 化学成分研究表明白苞裸蒴主要含有 黄酮类和木脂素类化合物 ${ }^{[3,4]}$. 现代药理研究显示白苍 裸蒴有抗菌、抗氧化和抑制 $\alpha$-葡萄糖苷酶等作用 ${ }^{[5,6]}$. 为 了进一步研究我国特有裸蒴属植物的化学成分, 发现结 构新颖有活性的先导化合物, 我们继续对采自四川峨眉
山地区的白苞裸龩进行了系统的化学成分的研究. 运用 中压硅胶柱色谱、MCI 凝胶柱色谱和高效液相制备色谱 等多种色谱技术从白苞裸蒴中分离纯化得到 6 个含氮类 化合物(图 1), 分别为 2-(1-差基-6-甲氧基-4-氧亚基环 己-2-烯-1-基)乙腈(1)、10-甲氧基-1-氧杂-3-氮杂螺[4.5] 癸-2,6-二烯-8-酮(2)、2-(1-羟基-4-氧亚基环己-2,5-二烯-

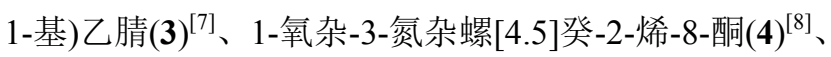

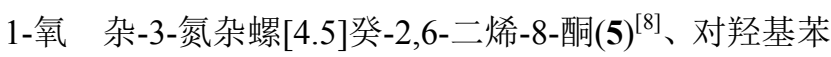
乙腈 $(6)^{[9]}$, 通过波谱技术鉴定了它们的结构. 其中化 合物 1 和 2 为新化合物, 化合物 3 为新的天然产物, 其 余化合物为首次从该植物中分离得到.

\footnotetext{
*E-mail: sjxiao@zmc.edu.cn

Received April 6, 2016; revised May 8, 2016; published online June 2, 2016.

Project supported by the National Natural Sciences Foundation of China (No. 31560102)

国家自然科学基金(No. 31560102)资助项目.
} 
<smiles>CO[C@H]1CC(=O)C=C[C@@]1(O)C#N</smiles><smiles>CO[C@H]1CC(O)C=CC12CN=CO2</smiles><smiles>COC1CCC2(CC1)CN=CO2</smiles><smiles>O=C1C=CC2(CC1)CN=CO2</smiles>

图 1 化合物 1 $\sim 6$ 的结构图

Figure 1 Structures of compounds $\mathbf{1} \sim \mathbf{6}$

\section{1 结果与讨论}

\section{1 化合物 1 的结构鉴定}

化合物 1 为浅黄色胶状物, HR-ESIMS 给出化合物 的分子式为 $\mathrm{C}_{9} \mathrm{H}_{11} \mathrm{NO}_{3}$ (测量值 $m / z 204.0630[\mathrm{M}+\mathrm{Na}]^{+}$, 计算值为 204.0631), 有 5 个不饱和度. ${ }^{1} \mathrm{H} \mathrm{NMR},{ }^{13} \mathrm{C}$ $\operatorname{NMR}$ (表 1)和 HSQC 谱图显示其有 1 个 $\alpha, \beta$ 不饱和羰基 单元 $\left[\delta_{\mathrm{H}} 6.78(\mathrm{~d}, J=10.2 \mathrm{~Hz}, 1 \mathrm{H}), 6.02(\mathrm{~d}, J=10.2 \mathrm{~Hz}\right.$, $\left.1 \mathrm{H}) ; \delta_{\mathrm{C}} 150.0,130.6,198.7\right], 1$ 个甲氧基 $\left[\delta_{\mathrm{H}} 3.45(\mathrm{~s}, 3 \mathrm{H})\right.$; $\left.\delta_{\mathrm{C}} 58.3\right], 2$ 个 $\mathrm{CH}_{2}\left[\delta_{\mathrm{H}} 2.63(\mathrm{dd}, J=16.9,7.6 \mathrm{~Hz}, 1 \mathrm{H}), 2.89\right.$ $(\mathrm{dd}, J=16.9,3.8 \mathrm{~Hz}, 1 \mathrm{H}), 2.86$ (d, $J=16.8 \mathrm{~Hz}, 1 \mathrm{H}), 2.94$ $\left.(\mathrm{d}, J=16.8 \mathrm{~Hz}, 1 \mathrm{H}) ; \delta_{\mathrm{C}} 39.6,26.3\right]$ 和 $\mathrm{OCH}\left[\delta_{\mathrm{H}} 3.77(\mathrm{dd}\right.$, $\left.J=7.6,3.8 \mathrm{~Hz}, 1 \mathrm{H}) ; \delta_{\mathrm{C}} 83.0\right], 1$ 个 $\mathrm{sp}^{3}$ 杂化的叔碳 $\left(\delta_{\mathrm{C}}\right.$ $71.4), 1$ 个 $\mathrm{sp}$ 杂化的碳 $\left(\delta_{\mathrm{C}} 118.4\right)$. 通过比较发现化合物 1 和 3 非常相似, 相比而言多了一个甲氧基, 少了一个不 饱和键. HMBC 谱中(图 2), H-6 和 C-2, C-1, C-2, C-4, $\mathrm{C}-\mathrm{OCH}_{3}$ 有相关, 甲氧基和 C-6 有相关, 说明甲氧基只能 处在 C-6 上. H-2 和 C-1, C-1, C-2, C-6 有相关, 可以确定 乙腈基处于 $\mathrm{C}-1$ 上. 至此确定了化合物的平面结构. NOESY 谱中, H-6 和 H-2 有相关, 说明 H-6 和乙腈基处 于同面, 进一步通过 H-6 的邻位偶合常数 $J=7.6,3.8 \mathrm{~Hz}$ 知 H-6 处于 $a$ 键, 乙腈基处于 $e$ 键, 即 H-6 和乙腈基处 于同面时构型较稳定, 此时乙腈基和甲氧基都可能处于 $e$ 键. 于是, 化合物 1 的相对构型得以确定, 鉴定为 2-(1羟基-6-甲氧基-4-氧亚基环己-2-烯-1-基)乙腈.

\section{2 化合物 2 的结构鉴定}

化合物 2 为浅黄色胶状物, HR-ESIMS 给出和化合 物 1 一样的分子式 $\mathrm{C}_{9} \mathrm{H}_{11} \mathrm{NO}_{3}$, 有 5 个不饱和度. ${ }^{1} \mathrm{H}$ $\mathrm{NMR},{ }^{13} \mathrm{C} \mathrm{NMR}$ (表 2)和 HSQC 谱图显示其和化合物 5 非常相似, 有 1 个噁唑啉单元 $\left[\delta_{\mathrm{H}} 7.29(\mathrm{t}, J=1.8 \mathrm{~Hz}, 1 \mathrm{H})\right.$, $3.53(\mathrm{dd}, J=17.9,1.8 \mathrm{~Hz}, 1 \mathrm{H}), 2.93(\mathrm{dd}, J=17.9,1.8 \mathrm{~Hz}$, $\left.1 \mathrm{H}) ; \delta_{\mathrm{C}} 147.3,41.5,85.2\right], 1$ 个 $\alpha, \beta$ 不饱和羰基 $\left[\delta_{\mathrm{H}} 6.82(\mathrm{~d}\right.$,
表 1 化合物 1 的 NMR 数据(400/100 MHz, $\left.\mathrm{CD}_{3} \mathrm{OD}\right)$

Table 1 NMR data of compound $1\left(400 / 100 \mathrm{MHz}, \mathrm{CD}_{3} \mathrm{OD}\right)$

\begin{tabular}{clc}
\hline No. & \multicolumn{1}{c}{$\delta_{\mathrm{H}}(J \mathrm{in} \mathrm{Hz})$} & $\delta_{\mathrm{C}}$ \\
\hline 1 & & 71.4 \\
2 & $6.78 \mathrm{~d}(10.2)$ & 150.0 \\
3 & $6.02 \mathrm{~d}(10.2)$ & 130.6 \\
4 & & 198.7 \\
5 & $2.63 \mathrm{dd}(16.9,7.6), 2.89 \mathrm{dd}(16.9,3.8)$ & 26.3 \\
6 & $3.77 \mathrm{dd}(7.6,3.8)$ & 83.0 \\
$\mathrm{CN}$ & & 118.4 \\
$\mathrm{CH}_{2}$ & $2.86 \mathrm{~d}(16.8), 2.94 \mathrm{~d}(16.8)$ & 39.6 \\
$\mathrm{OCH}_{3}$ & $3.45 \mathrm{~s}$ & 58.3 \\
\hline
\end{tabular}

$J=10.1 \mathrm{~Hz}, 1 \mathrm{H}), 5.94(\mathrm{~d}, J=10.1 \mathrm{~Hz}, 1 \mathrm{H}) ; \delta_{\mathrm{C}} 149.6$, $129.8,196.6], 1$ 个甲氧基 $\left[\delta_{\mathrm{H}} 3.42(3 \mathrm{H}, \mathrm{s}) ; \delta_{\mathrm{C}} 58.4\right], 1$ 个 $\mathrm{sp}^{3}$ 杂化的叔碳 $\left(\delta_{\mathrm{C}} 85.2\right), 1$ 个 $\mathrm{CH}_{2}\left[\delta_{\mathrm{H}} 2.76(\mathrm{dd}, J=16.4\right.$, $\left.4.3 \mathrm{~Hz}, 1 \mathrm{H}), 2.47(\mathrm{dd}, J=16.4,10.3 \mathrm{~Hz}, 1 \mathrm{H}) ; \delta_{\mathrm{C}} 41.1\right]$ 和 OCH $\left[\delta_{\mathrm{H}} 3.86(\mathrm{dd}, J=10.3,4.4 \mathrm{~Hz}, 1 \mathrm{H}) ; \delta_{\mathrm{C}} 79.7\right]$. HMBC 谱中(图 2), H-10 和 C-6 有相关, 说明甲氧基只能处在 C-10 上; H-4 和 C-5, C-6, C-10 有相关可以确定螺环的存 在. 通过 $\mathrm{H}-10\left[\delta_{\mathrm{H}} 3.86(\mathrm{dd}, J=10.3,4.4 \mathrm{~Hz}, 1 \mathrm{H}) ; \delta_{\mathrm{C}} 79.7\right]$ 的偶合常数知道 H-10 处于坚直键, 通过和文献[8]中化 合物对比知道甲氧基处于 $\alpha$ 位. 于是, 化合物 $\mathbf{1}$ 的相对 构型得以确定，鉴定为 10-甲氧基-1-氧杂-3-氮杂螺[4.5] 癸-2,6-二烯-8-酮.

表 2 化合物 2 的 NMR 数据(400/100 MHz, $\left.\mathrm{CD}_{3} \mathrm{COCD}_{3}\right)$ Table 2 NMR data of compound $2\left(400 / 100 \mathrm{MHz}, \mathrm{CD}_{3} \mathrm{COCD}_{3}\right)$

\begin{tabular}{clc}
\hline No. & \multicolumn{1}{c}{$\delta_{\mathrm{H}}(J \mathrm{in} \mathrm{Hz})$} & $\delta_{\mathrm{C}}$ \\
\hline 2 & $7.29 \mathrm{t}(1.8)$ & 147.3 \\
4 & $3.53 \mathrm{dd}(17.9,1.8), 2.93 \mathrm{dd}(17.9,1.8)$ & 41.5 \\
5 & & 85.2 \\
6 & $6.82 \mathrm{~d}(10.1)$ & 149.6 \\
7 & $5.94 \mathrm{~d}(10.1)$ & 129.8 \\
8 & & 196.6 \\
9 & $2.76 \mathrm{dd}(16.4,4.3), 2.47 \mathrm{dd}(16.4,10.3)$ & 41.1 \\
10 & $3.86 \mathrm{dd}(10.3,4.3)$ & 79.7 \\
$\mathrm{OCH}_{3}$ & $3.42 \mathrm{~s}$ & 58.4 \\
\hline
\end{tabular}

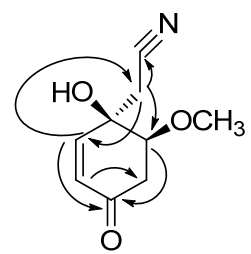

1

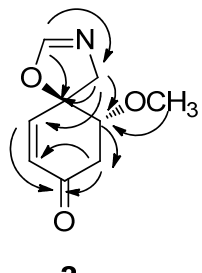

2
图 2 化合物 1 和 $\mathbf{2}$ 的主要的 HMBC ( $\frown$ )相关 Figure 2 Key HMBC ( $\frown$ ) correlations of compounds $\mathbf{1}$ and $\mathbf{2}$

\section{2 实验部分}

\section{1 仪器与试剂}

质谱用 Finnigan $\mathrm{LCQ}^{\mathrm{DECA}}$ 型质谱仪和 Bruker Bi- 
OTOF Q 型质谱仪测定; 核磁共振用 Bruker BiOTOF Q 型核磁共振仪测定, TMS 为内标; IR 用 FTIR-850 傅里叶 变换红外光谱仪, $\mathrm{KBr}$ 压片. 薄层色谱硅胶 $\mathrm{GF}_{254}$ 和柱色 谱硅胶(300 400 目)均为青岛海洋化工厂生产; MCI 凝 胶为 Mitsubishi Chemical MCI GEL CHP20/P120 (37 $75 \mu \mathrm{m})$; 半制备高效液相色谱仪用北京创新通恒 LC3000 型色谱仪, HPLC 柱用 Kromasil $\mathrm{C}_{18}, 5 \mu \mathrm{m}, 10$ $\mathrm{mm} \times 250 \mathrm{~mm}$.

\section{2 植物材料}

白苞裸蒴全草 2013 年 10 月采自四川峨眉山, 由峨 眉山市成都中医药大学峨眉学院(原四川省中药学校) 祝世杰研究员鉴定为白苞裸蒴(Gymnotheca involucrata Pei), 样品标本(No.20141011)保存在中国科学院成都生 物研究所天然产物中心.

\section{3 提取分离}

白苞裸蒴全草干重 $2.6 \mathrm{~kg}$ 粉碎后用甲醇浸泡提取 3 次, 减压浓缩得浸膏 $374 \mathrm{~g}$, 将其分散于热水 $(2.0 \mathrm{~L})$ 中用 石油醚 $(2.0 \mathrm{~L} \times 3)$ 、乙酸乙酯 $(2.0 \mathrm{~L} \times 3)$ 和正丁醇 $(2.0 \mathrm{~L} \times$ 3)依次萃取, 减压浓缩各提取液得石油醚浸膏 $60 \mathrm{~g}$, 乙 酸乙酯部位浸膏 $31 \mathrm{~g}$. 乙酸乙酯部位浸膏经 MCI柱色谱 $[V$ (甲醇) $: V($ 水 $)=9: 1$ ] 洗脱, 浓缩洗脱液得浸膏 $18 \mathrm{~g}$. 该浸膏经中压硅胶柱色谱(石油醚一丙酮梯度洗脱 100 ： $1 \rightarrow 1: 100$ )分为 6 个部分 Fr.1 Fr.6. 第二部分 Fr.2 经中 压硅胶柱(石油醚一丙酮 $20: 1 \rightarrow 1: 1$ )分为 7 个部分 Fr.2.1 Fr.2.7. Fr.2.6 经半制备 HPLC 分离 $[V(\mathrm{MeOH})$ : $V($ 水 $)=65: 35,3.0 \mathrm{~mL} / \mathrm{min}$ ] 得到 Fr.2.6A; Fr.2.6A 进一步 经半制备 HPLC 分离 $\left[V(\mathrm{MeOH}): V\left(\mathrm{H}_{2} \mathrm{O}\right)=60: 40,3.0\right.$ $\mathrm{mL} / \mathrm{min}$ ]得到化合物 $2(t=10.7 \mathrm{~min}, 9 \mathrm{mg})$. 第三部分 Fr.3 经中压硅胶柱 (石油醚-丙酮 $50: 1 \rightarrow 1: 5$ )分为 6 个 部分 Fr.3.1 Fr.3.6. Fr.3.1 经半制备 HPLC 分离 $\left[V(\mathrm{MeOH}): V\left(\mathrm{H}_{2} \mathrm{O}\right)=65: 35,3.0 \mathrm{~mL} / \mathrm{min}\right]$ 得到 Fr.3.1B, Fr.3.1B 进一步经半制备 HPLC 分离 $[V(\mathrm{MeOH})$ : $V\left(\mathrm{H}_{2} \mathrm{O}\right)=45: 55,2.0 \mathrm{~mL} / \mathrm{min}$ ]得到化合物 $4(t=31.7$ $\mathrm{min}, 18 \mathrm{mg}$ ). Fr.3.3 经制备 HPLC 分离(甲醇-水梯度 40: $60 \rightarrow 95: 5$ ) 得到 3 个亚组分 Fr.3.3A Fr.3.3C; 亚组分 Fr.3.3A 进一步经半制备 HPLC 分离 $[V(\mathrm{MeOH})$ : $V\left(\mathrm{H}_{2} \mathrm{O}\right)=50: 50,2.0 \mathrm{~mL} / \mathrm{min}$ ]得到化合物 $\mathbf{5}(t=8.8 \mathrm{~min}$, $20 \mathrm{mg}), \mathbf{6}(t=13.3 \mathrm{~min}, 25 \mathrm{mg}), 3(t=15.8 \mathrm{~min}, 10 \mathrm{mg})$ 和 $1(t=18.7 \mathrm{~min}, 6 \mathrm{mg})$.

2-(1-羟基-6-甲氧基-4-氧亚基环己-2-烯-1-基)乙腈 (1): 浅黄色胶状物, $[\alpha]_{\mathrm{D}}^{20}+6(c 0.18, \mathrm{MeOH}) ;{ }^{1} \mathrm{H}$ NMR 和 ${ }^{13} \mathrm{C}$ NMR 数据见表 $1 ; \mathrm{UV}(\mathrm{MeOH}) \lambda_{\max }[\log \varepsilon /(\mathrm{L} \cdot$ $\left.\mathrm{mol}^{-1} \cdot \mathrm{cm}^{-1}\right)$ ]: $225(3.16) \mathrm{nm}$; IR (KBr) v: 2958, 1679, 1648, 1422, 1358, 1266, 1180, $1101 \mathrm{~cm}^{-1}$; ESIMS $\mathrm{m} / z$ : $204[\mathrm{M}+\mathrm{Na}]^{+}$; HR-ESIMS calcd for $\mathrm{C}_{9} \mathrm{H}_{11} \mathrm{NO}_{3} \mathrm{Na}[\mathrm{M}+$ $\mathrm{Na}]^{+}$204.0631, found 204.0630.

10-甲氧基-1-氧杂-3-氮杂螺 [4.5]癸-2,6-二烯-8-酮 (2): 浅黄色胶状物, $[\alpha]_{\mathrm{D}}^{20}-4(c 0.15, \mathrm{MeOH}) ;{ }^{1} \mathrm{H}$ NMR 和 ${ }^{13} \mathrm{C}$ NMR 数据见表 $2 ; \mathrm{UV}(\mathrm{MeOH}) \lambda_{\max }[\log \varepsilon /(\mathrm{L} \cdot$ $\mathrm{mol}^{-1} \cdot \mathrm{cm}^{-1}$ )]: $227(3.06) \mathrm{nm}$; IR (KBr) $v: 2938,1689$, $1608,1425,1379,1283,1182,1107,983,869,829 \mathrm{~cm}^{-1}$; ESIMS $m / z: 204[\mathrm{M}+\mathrm{Na}]^{+}$, HR-ESIMS calcd for $\mathrm{C}_{9} \mathrm{H}_{11} \mathrm{NO}_{3} \mathrm{Na}[\mathrm{M}+\mathrm{Na}]^{+}$204.0631, found 204.0630.

2-(1-羟基-4-氧亚基环己-2,5-二烯-1-基)乙腈(3)：白 色粉末. ${ }^{1} \mathrm{H}$ NMR $\left(400 \mathrm{MHz}, \mathrm{CDCl}_{3}\right) \delta: 6.91$ (d, $J=10.0$ Hz, 2H, H-2,5), 6.26 (d, $J=10.0 \mathrm{~Hz}, 2 \mathrm{H}, \mathrm{H}-3,4), 3.73$ (s, $1 \mathrm{H}, \mathrm{OH}), 2.77$ (s, 2H, H-7); ${ }^{13} \mathrm{C}$ NMR $\left(100 \mathrm{MHz}, \mathrm{CDCl}_{3}\right)$ $\delta: 184.9$ (C-4), 149.6 (C-2,5), 129.4 (C-3,4), 116.8 (C-1), 67.5 (C-2); ESI-MS m/z: $172[\mathrm{M}+\mathrm{Na}]^{+}$.

辅助材料(Supporting Information) 新化合物 1 和 $\mathbf{2}$ 的 HR-ESIMS 谱, 1D NMR 和 2D NMR 谱图. 这些材料可 以免费从本刊网站(http://sioc-journal.cn/)上下载.

\section{References}

[1] Chen, Y. Q. Flora of China, Vol. 20(1), Science Press, Beijing, 1982 (in Chinese).

(程用谦, 中国植物志, 第 20(1)卷, 科学出版社, 北京, 1982.)

[2] Zhang, H. Y.; Zhang, Z. Y. The Chinese Traditional Medicine Resource Records, Science Press, Beijing, 1994 (in Chinese). (张惠源, 张志英, 中国中药资源志要, 科学出版社, 北京, 1994.)

[3] Yang, W. L.; Tian, J.; Ding, L. S. Chin. J. Chin. Mater. Med. 2001, 26, 43 (in Chinese). (杨维力, 田军, 丁立生, 中国中药杂志, 2001, 26, 43.)

[4] Xiao, S. J.; Lei, X. X.; Xia, B.; Xu, D. Q.; Xiao, H. P.; Xu, H. X.; Chen, F.; Ding, L. S.; Zhou, Y. Tetrahedron. Lett. 2014, 55, 5949.

[5] Chen, B. Q.; Yang, Z. B.; Cao, N. F.; Zhao, L.; Kan, W. Y. Chin. Tradit. Pat. Med. 2011, 33, 677 (in Chinese).

(陈百泉, 杨再波, 曹乃锋, 赵琳, 康文艺, 中成药, 2011, 33, 677.)

[6] Yang, Z. B.; Long, C. M.; Mao, H. L.; Sun, C. B. Food Ind. 2011, 4, 79 (in Chinese).

(杨再波, 龙成梅, 毛海立, 孙成斌, 食品工业, 2011, 4, 79.)

[7] Fischer, A.; Henderson, G. N. Tetrahedron Lett. 1983, 24, 131.

[8] Xiao, S. J.; Guo, D. L; Chen, F.; Ding, L. S.; Zhou, Y. J. Asian Nat. Prod. Res. 2016, 18, 719.

[9] Yao, S. Y.; Ma, Y. B.; Tang, Y.; Chen, J. J.; Zhang, X. M. Chin. J. Chin. Mater. Med. 2008, 33, 1418 (in Chinese).

(姚淑英, 马云保, 唐亚, 陈纪军, 张雪梅, 中国中药杂志, 2008, 33,1418 .) 